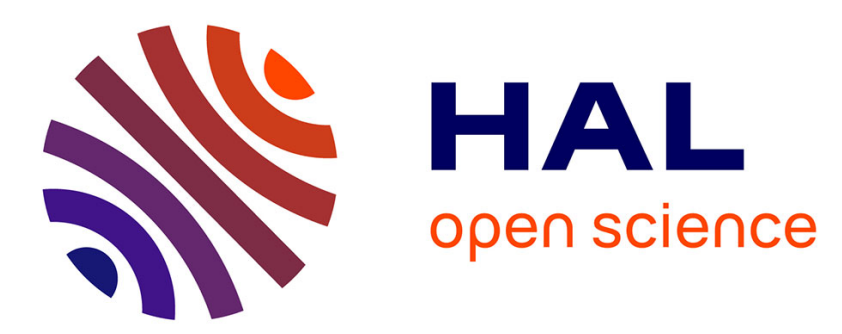

\title{
Analyse des performances de résolution de problèmes arithmétiques verbaux en début de collège
}

Annette Jarlegan, Catherine Billard, Youssef Tazouti, Catherine Thevenot, Michel Fayol

\section{- To cite this version:}

Annette Jarlegan, Catherine Billard, Youssef Tazouti, Catherine Thevenot, Michel Fayol. Analyse des performances de résolution de problèmes arithmétiques verbaux en début de collège. Annee Psychologique, 2020, 120 (4), pp.271-296. 10.3917/anpsy1.204.0271 . hal-03009560

\section{HAL Id: hal-03009560 https://hal.science/hal-03009560}

Submitted on 17 Nov 2020

HAL is a multi-disciplinary open access archive for the deposit and dissemination of scientific research documents, whether they are published or not. The documents may come from teaching and research institutions in France or abroad, or from public or private research centers.
L'archive ouverte pluridisciplinaire HAL, est destinée au dépôt et à la diffusion de documents scientifiques de niveau recherche, publiés ou non, émanant des établissements d'enseignement et de recherche français ou étrangers, des laboratoires publics ou privés. 
Titre : Analyse des performances de résolution de problèmes arithmétiques verbaux en début de collège

Annette Jarlégan, Université de Lorraine (LISEC, EA 2310), France

Catherine Billard, Centre de Référence sur les Troubles des Apprentissages, Hôpital Bicêtre, Paris, France

Youssef Tazouti, Université de Lorraine (2LPN, EA 7489), France

Catherine Thevenot, Université de Lausanne, Institut de Psychologie, Suisse

Michel Fayol, Université de Clermont Auvergne (LAPSCO, UMR 6024 UCA-CNRS), France 
RESUME : Cet article se propose d'étudier les performances d'élèves scolarisés au début du collège (en sixième et cinquième) à une large série de problèmes arithmétiques ou logiques. Par le biais d'indices socio-démographiques et d'épreuves testant des capacités soit générales (langage, mémoire à court terme, etc.) soit spécifiquement liées au domaine numérique, il cherche à identifier les variables ayant ou non une contribution significative sur les scores en résolution de problèmes et à déterminer leurs poids relatifs.

L'échantillon est constitué de 269 collégiens (129 en $6^{\text {ème }}$ et 140 en $5^{\text {ème) }}$, scolarisés dans des établissements scolaires, publics ou privés, choisis pour rendre compte d'une diversité de contextes sociaux ainsi que géographiques. Les résultats de la régression multiple hiérarchique mettent en évidence l'importance à ce niveau des savoirs et savoir-faire spécifiquement mathématiques, plus que celle des capacités générales ou des dimensions démographiques.

Mots-clés : apprentissages, mathématiques, problème arithmétique, capacités générales, collège.

\section{Abstract: Analyzing arithmetic word problem solving performance in middle school students.}

In this article, we study the performance of students in early sixth and seventh grade in a wide range of arithmetic or logical problems. By means of socio-demographic indexes and tests measuring abilities that are either general (language, short-term memory, etc.) or specifically related to the digital domain, we try to identify the variables with or without a significant contribution to the problem solving scores and to determine their relative weights.

The sample consists of 269 middle school students (129 in the 6th and 140 in the 7 th year), enrolled in public or private schools, selected to reflect a diversity of social and geographical contexts. The results of multiple hierarchical regression highlight the importance at this level of specifically mathematical knowledge more than that of general abilities or even demographic dimensions. 
Keywords: learning, mathematics, arithmetic problem, general abilities, middle school

\section{INTRODUCTION}

Un problème arithmétique verbal décrit brièvement une situation sous la forme d'une petite histoire. La réponse numérique devant être fournie à l'issue de sa résolution est obtenue par la réalisation d'une ou plusieurs opérations arithmétiques utilisant les données du texte. Un problème arithmétique verbal simple et classique est par exemple « Mike et Nath ont 8 billes ensemble. Nath a 5 billes. Combien Mike a-t-il de billes ? ». Le traitement de ce type de problèmes occupe une place importante dans les cursus scolaires. Il correspond à une activité très souvent sollicitée, notamment en vue d'évaluer les performances des élèves, comme en attestent les évaluations et comparaisons internationales PISA $2015^{1}$ (OECD, 2015) et TIMSS $2015^{2}$ (IEA, 2015). Il constitue aussi une source d'échecs, quel que soit le niveau. Il est donc important de chercher à comprendre l'origine des difficultés rencontrées par les élèves, avec comme objectif le souci d'intervenir en vue de prévenir ces difficultés ou d'équiper les élèves de connaissances et de savoir-faire susceptibles de leur permettre de les surmonter.

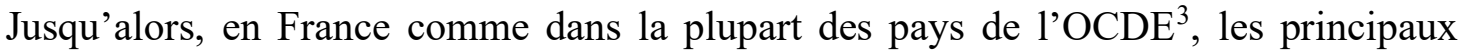
travaux relatifs à la résolution de problèmes arithmétiques verbaux ont porté sur la scolarité élémentaire (e.g., Carpenter, Hiebert, \& Moser, 1983 ; De Corte \& Verschaffel, 1987 ; Swanson, 2011 ; pour le cours préparatoire (Grade 1 dans les pays anglo-saxons); Fischer et al. (2019) ; Jordan \& Hanich, 2000 ; Wang, Fuchs \& Fuchs, 2016 ; Willis \& Fuson, 1988 pour le cours élémentaire 1 (Grade 2); Fuchs et al., 2006 ; Fuchs, et al., 2008 ; Rosenthal \& Resnick, 1974 ; Tarim, 2017 ; pour le cours élémentaire 2 ou Grade 3 ; pour une synthèse voir Fayol, Thevenot \& Devidal, 2005 ou Thevenot, 2017). Les élèves y découvrent ce type de problèmes, leurs structures et les situations qu'ils décrivent. Ils apprennent aussi à les traiter en s'appuyant

\footnotetext{
${ }^{1}$ Programme International pour le Suivi des Acquis des élèves

${ }^{2}$ Trends in International Mathematics and Science Study

${ }^{3}$ Organisation de coopération et de développement économiques
} 
soit sur des schémas de problèmes permettant d'apparier les composantes des problèmes à des structures stockées en mémoire à long-terme représentant les ensembles et leurs relations en vue de sélectionner les opérations (Dixon, 1987 ; Fayol, Abdi, \& Gombert, 1987 ; Kintsch \& Greeno, 1985), soit sur des modèles de situation (ou modèles mentaux) qui consistent en des simulations mentales en mémoire de travail des situations décrites (De Corte, Verschaffel, \& De Win, 1985; Thevenot, 2008; Thevenot \& Barrouillet, 2015; Thevenot, Devidal, Barrouillet, \& Fayol, 2007). Les modèles de situation semblent privilégiés par les enfants et les adultes lorsque la situation est facile à modéliser ou à imager mentalement (Brissiaud \& Sander, 2010 ; Bagnoud, Burra, Oakhill, \& Thevenot, 2018). La théorie des modèles de situation permet d'expliquer pourquoi les stratégies des enfants sont très influencées par des éléments contextuels des problèmes (e.g., Coquin-Viennot \& Moreau, 2003 ; Gamo, Taabane, \& Sander, 2011).

$\mathrm{Si}$, comme nous venons de le voir, de nombreux travaux portent sur les élèves du primaire, beaucoup moins de données sont en revanche disponibles pour la période immédiatement suivante, pourtant essentielle car elle correspond aux premières étapes du collège (voir Kail \& Hall, 1999 et Muth, 1994 chez des élèves de classe de sixième). À cette période de leur scolarité, les élèves vont devoir affronter des problèmes arithmétiques verbaux dont le texte décrira des situations portant sur de nouveaux domaines disciplinaires comme la physique, la biologie ou la géographie.

À l'entrée au collège, les élèves ont déjà développé une certaine expertise en résolution de problèmes arithmétiques verbaux : ils ont mémorisé les structures les plus fréquentes, acquis le lexique usuel des énoncés, appris à déterminer les procédures les plus souvent mobilisées ; ils connaissent aussi mieux les faits arithmétiques et les opérations. Ces acquis facilitent la résolution des problèmes (Muth, 1994). D'autres savoirs ou savoir-faire la rendent plus complexe : par exemple, les traitements portent désormais souvent sur des nombres rationnels 
dont la maîtrise est encore problématique (Lortie-Forgues, Tian, \& Siegler, 2015). Les difficultés auxquelles doivent faire face les élèves de classes de sixième et de cinquième de collèges peuvent donc différer de celles relevées chez leurs cadets, ce qui rend nécessaire leur exploration.

Dans ce cadre, l'objectif du présent article est double. Tout d'abord, il s'agit d'évaluer les performances d'élèves de sixième et de cinquième à une série de brefs problèmes arithmétiques verbaux présentés oralement et renvoyant à des situations mathématiques variées allant de déductions logiques n'impliquant pas de calculs à des situations comportant des données numériques nécessitant des traitements plus ou moins complexes portant sur des nombres naturels ou rationnels. Les problèmes ont été élaborés et testés par Ménissier (2011). Ensuite, il s'agit de chercher à identifier les origines des difficultés en étudiant l'impact des variables dont les effets sont classiquement mis en évidence (Kail, \& Hall, 1999 ; Muth, 1984 ; Swanson, Cooney, \& Bock, 1993 ; Tolar, Fuchs, Cirino, Fuchs, Hamlett, \& Fletcher, 2012): caractéristiques démographiques ou socio-culturelles des élèves (e.g. professions des parents ; Arnold \& Doctoroff, 2003; Bradley \& Corwyn, 2002) ; difficultés spécifiques aux mathématiques (e.g. les fractions ou les proportions; Siegler \& Pike, 2013 ; Van Dooren, Lehtinen, \& Verschaffel, 2015); capacités cognitives générales (e.g. attention; Swanson, 2011 ; Swanson, Olide, \& Kong, 2018) ; capacités langagières (e.g. niveau en vocabulaire ; Cummins, Kintsch, Reusser, \& Weimer, 1988 ; Davis-Dorsey, Ross, \& Morrison, 1991 ; Lewis \& Mayer, 1987).

\section{La résolution de problèmes arithmétiques verbaux}

Les recherches conduites au cours des trois dernières décennies ont montré que les difficultés majeures des activités de résolution de problèmes arithmétiques verbaux résident non pas, ou en tout cas principalement, dans les traitements numériques, même s'ils ont une 
certaine importance, mais dans la compréhension/interprétation des énoncés et dans la mise en relation du résultat de cette compréhension avec les procédures de résolution (Fayol et al., 2005).

Les énoncés relèvent à la fois du narratif puisqu'ils relatent une histoire, même si elle est extrêmement sommaire, et d'un type textuel particulier : le problème. Des informations manquent et l'élève doit pouvoir les calculer en s'appuyant sur les données fournies par l'énoncé et sur ses savoirs et savoir-faire précédemment acquis. Par ailleurs, la résolution doit faire appel au symbolisme mathématique (e.g., chiffres, signes) et aux traitements qui leur sont associés (e.g. opérations). Ce double statut permet de comprendre au moins partiellement les difficultés que soulève cette activité. Afin de déterminer la ou les opérations qui doivent être effectuées pour résoudre le problème, les enfants doivent préalablement comprendre la situation problème décrite par l'énoncé. La nature des inconnus et les relations entretenues par les inconnus et les différentes valeurs sont en effet à appréhender à partir du texte du problème. Comme déjà évoqué, cette étape de compréhension nécessite souvent de construire une représentation mentale la plus proche possible de celle qui est requise pour la résolution, en imageant et simulant mentalement les relations décrites par le texte (modèle mental, JohnsonLaird, 1983, Thevenot, 2010, ou modèle de situation, Brissiaud \& Sander, 2010 ; van Dijk \& Kintsch, 1983). Cependant, les enfants et les adultes peuvent parfois activer des représentations « toutes faites » en se basant sur des indices superficiels des problèmes, principalement sur les expressions mathématiques rencontrées fréquemment. Le terme « ensemble» dans un problème peut ainsi activer un schéma de combinaison et une procédure impliquant l'addition des termes du problème (Kintsch \& Greeno, 1985). L'utilisation de tels schémas de problèmes peut s'avérer très efficace lorsque les problèmes restent prototypiques mais peut également mener à des erreurs d'interprétation des problèmes lorsqu'une analyse moins superficielle des 
textes s'avère nécessaire (Lewis \& Mayer, 1987 ; Verschaffel, De Corte, \& Lasure, 1994 ; Jimenez \& Verschaffel, 2014, voir Thevenot, 2017 pour une synthèse).

Plusieurs résultats ont montré que les élèves issus de milieux défavorisés éprouvent des difficultés lors de l'étape de construction d'une représentation mentale des problèmes arithmétiques verbaux à partir du texte. D’une part, les univers de référence décrits leur sont moins familiers qu'aux élèves plus favorisés (Stern \& Lehrndorfer, 1992); d'autre part, leur maîtrise du langage est moindre ou moins habile, ce qui peut conduire à des interprétations erronées. Levine, Jordan et Huttenlocher (1992) ont montré que les difficultés associées à la présentation verbale des informations commencent très tôt. Ces auteurs ont comparé chez des enfants de quatre à six ans la résolution de problèmes additifs et soustractifs fournis soit sous forme de manipulations d'éléments sans verbalisation (e.g. balles qui sont placées dans une boîte opaque par exemple) soit sous forme d'énoncés verbaux (e.g., Michel avait x balles. Il en reçoit y de plus. Combien a-t-il de balles maintenant?). Les performances étaient meilleures avec les problèmes présentés sous forme non verbale qu'avec ceux qui étaient formulés verbalement. Dès 4 ans, les enfants réussissaient au moins certains items sous leur forme verbale mais il fallait attendre qu'ils aient $51 / 2$ à 6 ans pour parvenir à des succès équivalents entre la présentation sous forme verbale et non-verbale. Jordan, Huttenlocher et Levine (1992) ont repris le même paradigme et comparé les performances d'enfants de maternelle issus de milieux favorisés ou défavorisés. Elles ont relevé une interaction entre la modalité de présentation et le statut social. Alors que les deux groupes d'enfants obtenaient les mêmes performances aux épreuves non verbales, les enfants de milieux favorisés réussissaient mieux que les autres avec la présentation verbale des situations. De plus, lorsque le niveau langagier était statistiquement contrôlé, les deux groupes faisaient jeu égal. Le suivi de la même population en Cours Préparatoire après un début d'enseignement formel fait apparaître des progrès des deux groupes sous la modalité de présentation verbale (mais pas sous la modalité 
non verbale) mais, en dépit d'une importante amélioration de leurs performances, les élèves issus de milieux défavorisés ne rattrapent pas ceux du groupe favorisé (Jordan, Levine, \& Huttenlocher, 1994). De là l'importance de prendre en considération l'origine sociale des élèves pour étudier les performances en résolution de problèmes arithmétiques verbaux.

Le recours au langage, parce qu'il exige que les individus puissent évoquer les quantités notamment à partir de leur dénomination verbale, rend ainsi plus difficile la résolution de problèmes arithmétiques verbaux (Schumacher \& Fuchs, 2012). À cela s'ajoutent les difficultés plus spécifiquement lexicales ou rhétoriques (Pimperton, \& Nation, 2010 ; Purpura \& Reid, 2016). De là l'importance de soumettre les élèves à des épreuves permettant d'évaluer leurs niveaux en vocabulaire et en compréhension puisque l'emploi d'un lexique adapté à la population testée améliore significativement les performances des enfants (De Corte \& Verschaffel, 1987; De Corte, Verschaffel \& De Win, 1985). Comme par ailleurs la présentation des énoncés sous format écrit conduit au cumul des difficultés langagières et de celles qui ressortissent à la lecture elle-même, le choix a été fait dans cette recherche de présenter oralement les énoncés des problèmes avec la possibilité pour l'enfant de relire l'énoncé, bien que cela ne garantisse que partiellement une meilleure réussite (Jordan, Kaplan \& Hanish, 2002).

La présentation orale des énoncés entraîne que les informations parviennent de manière séquentielle à l'élève qui doit à la fois mobiliser son attention et ses capacités d'inhibition pour éviter d'en encoder certaines, les stocker temporairement, notamment celles qui ont trait aux données numériques, élaborer en temps réel ou plus tard une représentation de la situation, déterminer quelles sont les inconnues et comment procéder pour les calculer et réaliser les calculs. Un éventuel contrôle final est également possible, qui nécessite la prise en compte de l'intégralité des informations. L'ensemble de ces opérations mentales exige que soient mobilisées les ressources en attention, en raisonnement et en fonctions exécutives, (Friso-van 
den Bos, van der Ven, Kroesbergen, \& Van Luit, 2013 ; Peng, Namkung, Barnes, \& Sun, 2016 ; Raghubar, Barnes, \& Hecht, 2010). Leur importance serait toutefois plus forte chez les élèves les plus jeunes du fait que l'expérience et l'instruction reçue conduisent à l'apprentissage de faits numériques permettant de soulager la mémoire de travail et à l'apprentissage de stratégies facilitant le traitement des données. Les savoirs et savoir-faire spécifiques liés à l'arithmétique joueraient donc un rôle de plus en plus important avec l'élévation du niveau scolaire, ce qui justifie l'utilisation d'épreuves spécifiquement destinées à évaluer les performances numériques (e.g. en utilisation du code verbal et du code indo-arabe en lecture, écriture et transcodages complexes ; Fayol, Barrouillet, \& Renaud, 1996 ; Jarlégan, Fayol, \& Barrouillet, 1996, et en calcul mental et en calcul écrit).

Au total, l'objectif du présent article est d'abord de présenter les performances d'élèves typiques scolarisés au début du collège (en sixième et cinquième) à une large série de problèmes arithmétiques verbaux, une information jusqu'alors non disponible en France relativement à cette période de la scolarité. Un second objectif consiste à rechercher par le biais d'épreuves testant leurs capacités soit générales (langage, attention, raisonnement) soit spécifiques au domaine du nombre (calcul mental, opérations), les dimensions susceptibles d'expliquer ces performances, et donc de permettre de cibler et guider les interventions ultérieures. Les données issues des travaux antérieurs permettent d'attendre encore à cette période de la scolarité un impact significatif du niveau socio-culturel des familles (SES) et en particulier une médiatisation de cet impact par le langage une fois contrôlées les performances aux épreuves générales non verbales (raisonnement, attention). Enfin, et en conformité avec les faits rapportés par Geary, Nicholas, Li, et Sun (2017), nous émettons l'hypothèse que la dimension mathématique présente un poids significatif et important à ce niveau de la scolarité une fois contrôlées les autres variables. Ce poids tiendrait à la spécificité des savoirs et savoir-faire 
mathématiques devant être mobilisés pour résoudre en classes de sixième et cinquième les problèmes arithmétiques verbaux.

\section{METHODE}

\subsection{Participants}

L'échantillon est constitué de 269 collégiens (129 en $6^{\text {ème }}$ et 140 en $5^{\text {ème) }}$, scolarisés dans des établissements scolaires publics ou privés, volontaires après information et accord des parents. Tous les enfants des classes retenues ont été examinés à l'exclusion des enfants bénéficiant d'une reconnaissance de handicap, ou dont aucun des deux parents ne parlait le français. Les établissements ont été choisis pour rendre compte d'une diversité de contextes sociaux ainsi que géographiques (Paris, Toulouse, Orléans, banlieue parisienne et territoire rural).

Le tableau I présente les principales caractéristiques de l'échantillon : sexe, bilinguisme des parents (\% d'enfants dont un des deux parents parle une autre langue que le français), suivi orthophonique en cours ou antérieur au collège et catégorie socioprofessionnelle des parents (calculée en attribuant à l'élève la catégorie socio-professionnelle (CSP) la plus élevée des deux parents selon la classification de l'INSEE (2003), puis recodée en trois catégories).

Tableau I : Caractéristiques de l'échantillon

Table I: Characteristics of the sample

\begin{tabular}{|c|c|c|}
\hline Classes & $6^{\text {ème }}$ & $5^{\text {ème }}$ \\
\hline Effectif & 129 & 140 \\
\hline Age moyen en années (mini-maxi) & $11,5(10-12,3)$ & $12,4(11,2-13,7)$ \\
\hline Garçons $(\%)$ & $66(51 \%)$ & $79(56 \%)$ \\
\hline Filles $(\%)$ & $63(49 \%)$ & $61(44 \%)$ \\
\hline \multicolumn{3}{|l|}{ CSP (\%) } \\
\hline $1=$ défavorisée & $34 \%$ & $31 \%$ \\
\hline $2=$ moyenne & $32 \%$ & $32 \%$ \\
\hline $3=$ favorisée & $34 \%$ & $37 \%$ \\
\hline Bilinguisme & $35 \%$ & $37 \%$ \\
\hline Suivi orthophonique & $16 \%$ & $16 \%$ \\
\hline
\end{tabular}




\subsection{Protocole}

Après que le protocole a été ajusté ${ }^{4}$ en fonction des résultats aux pré-tests effectués en mai 2015 sur un échantillon de 24 jeunes de $6^{\text {ème }}$ et $5^{\text {ème }}$ sélectionnés par leur enseignant pour leur bon niveau scolaire, une version informatisée en a été tirée.

Huit étudiants en orthophonie et une neuropsychologue ont réalisé les passations après deux séances d'entrainement collectif dans les mêmes conditions que l'expérimentation (consignes, ordre de passation, cahier de réponses, etc.). Les passations se sont déroulées pendant l'année scolaire 2015-2016 avec environ un tiers des enfants examinés chaque trimestre. Trois séances d'une heure maximum, dont une consacrée aux épreuves de cognition numérique, espacées de moins de 15 jours, étaient effectuées sur le temps scolaire et parascolaire. L'ensemble des épreuves a été administré dans l'ordre suivant : langage écrit, cognition numérique et capacités générales.

Les passations ont été réalisées via un site internet sécurisé sur une tablette surface pro 3 sous windows 8. Afin d'assurer l'homogénéisation des passations, les items qui nécessitaient d'être énoncés verbalement à l'enfant avaient été préalablement enregistrés et étaient énoncés par l'application. Les réponses de l'enfant étaient soit enregistrées automatiquement pour les épreuves avec réponse tactile, soit enregistrées directement par l'examinateur ou colligées sur la feuille de passation puis reportées a posteriori sur l'application à partir d'une feuille de cotation explicite.

\footnotetext{
${ }^{4}$ Le protocole a été ajusté de manière à supprimer des items ayant entraîné un pourcentage d'échec trop important au pré-test et à modifier l'ordre de présentation des problèmes.
} 


\subsection{Mesures}

\subsubsection{Les épreuves de cognition numérique}

Elles évaluent différents domaines du calcul et de la construction du nombre : système numérique et la résolution de problèmes.

- Le système numérique était évalué par des épreuves testant les compétences informelles (chaîne verbale) et formelles (transcodages et base 10) (Jarlégan et al., 1996).

- La chaine verbale à rebours de 3 en 3 du nombre 420 jusqu'à 390 cotée automatiquement en 1 (juste) ou 0 (fausse)

- La dictée de 12 nombres comportant de grands nombres (506037) et des nombres décimaux (trois et huit centièmes), cotée en bonnes réponses ( 0 à12).

○ La lecture de 9 grands nombres (7000132), cotée en bonnes réponses ( 0 à 9 ).

○ Une épreuve de transcodage : l'application dictait successivement 5 nombres et l'enfant devait identifier le nombre écrit en code arabe parmi 3 autres distracteurs. Exemple : nombre-cible : « quarante-sept mille huit cent treize » à sélectionner parmi : 4700813 - 407813 - 47813 - 478013. La cotation était automatique en bonnes réponses (0 à 5).

○ Une épreuve testant les compétences en base $10: 1$ 'enfant devait résoudre par écrit une série de 10 opérations (additions, soustractions, multiplications ou divisions) présentées sur l'application en ligne, sans les poser, en mobilisant sa compréhension du système de la numération en base 10 (Exemple : 0,10 +0,2 $=\ldots$. . La cotation était en bonnes réponses $(0$ à 10$)$.

- Le calcul était évalué par une épreuve de fluence du calcul mental écrit, une épreuve de calcul mental avec lexique oral et 3 opérations à poser. 
○ L'évaluation du calcul mental écrit s'inspirait du modèle du test Hollandais Tempo Test Rekenen (TTR, de Vos, 1992). Pour explorer la fluence arithmétique et le calcul mental complexe, 5 séries de 40 calculs -additions, soustractions, multiplications, divisions et opérations mélangées- étaient proposées à l'élève sur support papier, sans le prévenir du type d'opération demandée. Il devait traiter le plus d'opérations possible de chaque série en 1 minute et disposait de 30 secondes de pause entre chaque série. La consigne stipulait qu'il n'avait pas le droit de compter sur ses doigts. Le score était le nombre de bonnes réponses sur 40 en 1 minute pour chacune des 5 opérations.

- Le calcul mental avec lexique oral concernait la résolution orale des 9 calculs formulés avec des termes lexicaux spécifiques aux mathématiques tels que dixième, quart, tiers, double, moitié. Exemple : le quart de 36 est égal à ? Le score était le nombre des bonnes réponses enregistrées automatiquement (0 à 9)

○ Trois opérations à poser : une addition, une soustraction et une multiplication comportant au moins un nombre décimal étaient présentées en ligne, l'enfant devait les poser et les résoudre. Le score de réussites était de 0 à 3.

\section{- La résolution de problèmes arithmétiques verbaux (cf. Annexe 2)}

L'épreuve comportait 20 problèmes de différents types : additifs, multiplicatifs, à étapes, etc. (Billard, Mirassou, \& Touzin, 2019 ; Ménissier, 2011). La tablette affichait et lisait l'énoncé de chaque problème. L'élève conservait l'énoncé du problème sous les yeux. Il disposait d'un brouillon pour ses calculs, et donnait sa réponse oralement à l'examinateur. Les problèmes étaient présentés selon un ordre de difficulté croissante. Le temps imparti pour résoudre le problème après qu'il avait été énoncé par l'application était limité à 1 
minute pour les plus faciles et à 2 minutes pour les 2 les plus difficiles. Le score total correspond au nombre de problèmes résolus correctement dans le temps imparti.

\subsubsection{Les épreuves évaluant les apprentissages en langage écrit et les compétences verbales}

- Les apprentissages en langage écrit étaient évalués par une épreuve de compréhension en lecture et une épreuve de dictée de texte (orthographe phonétique, lexicale et grammaticale), créées spécifiquement pour une batterie récemment commercialisée (Billard, Mirassou, Touzin, 2019). L'épreuve de compréhension en lecture comportait 13 questions sur un texte de 540 mot adaptés aux classes de 6-5 ${ }^{\text {ème }}$. Les treize questions en lecture concernaient soit des rappels de faits (par exemple "Que doivent préparer les élèves pour la fête de la musique?»), soit des inférences (par exemple : «Comment se sent-elle pendant la fête de la musique? Qu'est-ce qui te le fait dire? »). La cotation de la compréhension est exprimée en nombre de bonnes réponses. En dictée, la transcription d'un texte de 63 mots permettait d'évaluer les aspects en orthographe phonétique, lexicale et grammaticale. Le score total de dictée est exprimé en erreurs.

- Les compétences verbales étaient évaluées par une épreuve d'évocation lexicale (dénomination de 40 images cotée en bonnes réponses selon un barème prédéfini) et une épreuve de compréhension lexicale qui consistait en la désignation de 33 mots énoncés par l'application parmi 5 distracteurs (avec enregistrement automatique des bonnes réponses). Par exemple, l'image correspondant au mot «chalutier» énoncé par l'application était proposée parmi 5 autres images représentant un fleuve, un voilier, un paquebot, une péniche ou des poissons. 


\section{- 2.3.3. Les épreuves évaluant les capacités générales}

Les capacités générales étaient évaluées par une épreuve de matrices et une épreuve d'attention auditive.

- L'épreuve de matrices était inspirée de celles de Raven. La matrice de quatre ou six cases, dont l'une vide, était présentée au centre de l'écran. En dessous, se situaient six propositions de réponses. L'enfant devait retrouver parmi celles-ci la solution de la matrice, laquelle était enregistrée automatiquement (score/24).

- L'attention auditive comportait une partie contrôle et une partie conflit. La partie contrôle était une tâche simple de type Go-no-go, qui faisait appel aux capacités d'attention sélective et d'inhibition. L'enfant devait appuyer sur le rond dès qu'il entendait le mot « rond » et ne pas produire de réponse lors de l'énonciation des autres mots. Dans la partie conflit l'enfant devait alterner entre plusieurs consignes et inhiber des réponses prépondérantes pour répondre à des consignes conflictuelles. Les variables retenues sont le nombre de bonnes réponses simples (appuyer sur la forme dictée) ainsi que le nombre de bonnes réponses de flexibilité (rond sur carré ou carré sur rond).

\section{RESULTATS}

\subsection{Analyses descriptives des différentes variables de l'étude}

Le tableau II présente les moyennes, les écarts-types et étendues des différentes variables de l'étude. Une analyse en composante principale a été effectuée sur les 20 problèmes de mathématiques. Elle a permis de retenir un seul facteur. Le coefficient de consistance interne (alpha de Cronbach) de l'ensemble de l'échelle est satisfaisant (alpha $=0,83)$. Nous avons ensuite construit un score composite à partir des 20 items.

Tableau II : Statistiques descriptives des différentes variables de l'étude

Table II : Descriptive statistics 


\begin{tabular}{lcccccc}
\hline & Moyenne & Ecart type & Min & Max & Skewness & Kurtosis \\
\hline Cognition numérique & & & & & &,- 726 \\
Problèmes & 10,04 & 4,49 & 0 & 19 &,- 049 &, 117 \\
Calcul mental écrit & 79,36 & 18,61 & 14 & 129 &, 067 &,- 058 \\
Dictée de nombres & 9,25 & 2,58 & 2 & 12 &,- 862 & $-1,514$ \\
Chaine verbale &, 33 & -- & 0 & 1 &, 705 & 3,835 \\
Lecture de nombres & 8,13 & 1,51 & 2 & 9 & $-2,038$ &,- 806 \\
Calcul mental avec lexique oral & 5,37 & 2,27 & 0 & 9 &,- 192 & 3,803 \\
Transcodage & 4,37 & 1,09 & 0 & 5 & $-2,026$ &,- 975 \\
Opérations & 1,59 &, 96 & 0 & 3 &,- 019 & $-1,204$ \\
Base 10 & 5,52 & 3,05 & 0 & 10 &,- 221 &,- 248 \\
\hline Langage & & & & & &,- 236 \\
Evocation lexicale & 28,56 & 6,46 & 11 & 44 &,- 287 &,- 762 \\
Compréhension en lecture & 12,71 & 3,86 & 2 & 21 &,- 354 & 1,638 \\
Dictée & 16,76 & 8,69 & 0 & 39 &, 184 &,- 389 \\
\hline Capacités générales & & & & & & $-1,217$ \\
Attention auditive & 33,96 & 4,37 & 15 & 40 &,- 361 & \\
Matrices & 24,47 & 4,56 & 12 & 34 & &
\end{tabular}

L'examen des indices de la normalité univariée des scores aux différentes épreuves, à savoir les coefficients d'asymétrie et d'aplatissement, ne révèle aucune violation de la normalité de leur distribution. En effet, les coefficients d'asymétrie sont tous inférieurs à 3.00, valeur absolue du seuil au-dessus de laquelle la symétrie est jugée problématique (Kline, 1998). Les coefficients d'aplatissement sont tous inférieurs à 10.00, valeur absolue du seuil au-dessus de laquelle la normalité de la distribution est jugée problématique (Kline, 1998).

\subsection{Modèles de régression hiérarchique}

Dans les analyses qui suivent (cf. Tableau III), les performances des élèves à l'épreuve de résolution de problèmes verbaux constituent la variable dépendante dont on cherche à expliquer la variabilité. Afin de déterminer les principaux prédicteurs de la réussite en résolution de problème arithmétique verbal, le traitement des données est réalisé au moyen d'analyses de régression hiérarchique. Nous procédons en différentes étapes, en introduisant successivement dans les modèles différents blocs de variables susceptibles de contribuer à l'explication de la variabilité des performances en résolution de problème. Pour contrôler l'effet des caractéristiques socio-démographiques sur le rendement en résolution de problème 
arithmétique verbal, les variables « sexe de l'élève », «CSP des parents » et « niveau de classe » ont été entrées dans le modèle de l'étape 1. Nous introduirons ensuite successivement les variables liées aux capacités générales (modèle 2), puis les variables liées aux épreuves de langage (modèle 3) et enfin celles liées à la cognition numérique (modèle 4).

Tableau III : Analyses de régression hiérarchique prédisant la performance en « Résolution de problèmes verbaux »

Table III : Hierarchical regression analyses predicting performance in « arithmetic word problems »

\begin{tabular}{|c|c|c|c|c|}
\hline \multirow[b]{2}{*}{ Variables indépendantes } & \multicolumn{4}{|c|}{ Score aux problèmes mathématiques } \\
\hline & Modèle 1 & Modèle 2 & Modèle 3 & Modèle 4 \\
\hline & $\beta$ & $\beta$ & $\beta$ & $\beta$ \\
\hline \multicolumn{5}{|l|}{ Etape 1} \\
\hline Sexe $(0=$ garçon $; 1=$ fille $)$ &,$- 16 * *$ &,$- 20 * *$ &,$- 28 * *$ &,- 04 \\
\hline \multicolumn{5}{|l|}{ CSP (modalité de référence : défavorisée) } \\
\hline moyenne & ,06 & ,03 &,- 01 & ,01 \\
\hline favorisée &, $31 * *$ & $16^{*}$ &, 06 & 001 \\
\hline Classe $\left(0=6^{\text {ème }} ; 1=5^{\text {ème }}\right)$ &,- 01 &,- 02 &,- 05 &,- 04 \\
\hline$\Delta \mathrm{R}^{2}$ &, 11 & & & \\
\hline $\mathrm{R}^{2}$ &, 11 & & & \\
\hline \multicolumn{5}{|l|}{ Variation de $F=7,53, p<.001$} \\
\hline \multicolumn{5}{|l|}{ Etape 2} \\
\hline Attention auditive & &, $13^{*}$ & ,07 &,- 02 \\
\hline Matrices & & $41 * *$ &, $17^{*}$ &, $16^{* *}$ \\
\hline$\Delta \mathrm{R}^{2}$ & & ,19 & & \\
\hline $\mathrm{R}^{2}$ & &, 30 & & \\
\hline \multicolumn{5}{|l|}{ Variation de $F=33,86, p<.001$} \\
\hline \multicolumn{5}{|l|}{ Etape 3} \\
\hline Evocation lexicale & & &, $22 * *$ & ,02 \\
\hline Compréhension en lecture & & &, $19 * *$ &, 10 \\
\hline Dictée & & &,$- 17 * * 5$ &, 09 \\
\hline$\Delta \mathrm{R}^{2}$ & & &, 11 & \\
\hline $\mathrm{R}^{2}$ & & & 41 & \\
\hline \multicolumn{5}{|l|}{ Variation de $F=14,81, p<.001$} \\
\hline \multicolumn{5}{|l|}{ Etape 4} \\
\hline Calcul mental écrit & & & &, $15 * *$ \\
\hline Dictée des nombres & & & &, 02 \\
\hline Chaine verbale & & & &, 06 \\
\hline Lecture nombres & & & &, $13 *$ \\
\hline Calcul mental avec lexique oral & & & &, $36 * *$ \\
\hline Transcodage & & & & ,02 \\
\hline Opérations & & & & ,03 \\
\hline Base 10 & & & &, $21 * *$ \\
\hline$\Delta \mathrm{R}^{2}$ & & & &, 29 \\
\hline $\mathrm{R}^{2}$ & & & &, 70 \\
\hline Variation de $\mathrm{F}=28,07, \mathrm{p}<.001$ & & & & \\
\hline
\end{tabular}

\footnotetext{
${ }^{5}$ La cotation de la dictée se faisant en nombre d'erreurs, il est normal d'obtenir un coefficient négatif pour cette
} épreuve. 
Notes : $-* \mathrm{p}<.05 ; * * \mathrm{p}<.01$. Les coefficients de régression standardisés sont reportés

- Multicolinéarité : la tolérance varie entre .34 et .99

Les résultats de la régression multiple hiérarchique (tableau 3) révèlent qu'une fois les variables socio-démographiques prises en compte (étape 1), le sexe de l'élève et la CSP des parents permettent de prédire les performances à l'épreuve de résolution de problèmes, avec des performances significativement meilleures pour les garçons et pour les élèves de CSP favorisée $^{6}$. La prise en compte des capacités générales (attention auditive et matrices) dans l'étape 2 augmente le pouvoir explicatif du modèle, l'attention auditive et l'épreuve de matrices contribuant toutes deux significativement à la prédiction des performances en résolution de problèmes $(\Delta \mathrm{R} 2=0,19$, Variation de $\mathrm{F}=33,86, \mathrm{p}<.001)$. L'introduction des variables liées aux compétences en langage (évocation lexicale, compréhension en lecture et dictée), dans l'étape 3, contribue également à augmenter le pourcentage de variance expliquée du modèle qui atteint alors $41 \%$. Les performances en évocation lexicale, en compréhension de lecture et en dictée contribuent significativement à la prédiction des performances en résolution de problèmes $(\Delta \mathrm{R} 2=0,11$, Variation de $\mathrm{F}=14,81, \mathrm{p}<.001)$. Notons qu'à cette étape, lorsque les compétences liées au langage sont introduites dans l'équation, les contributions de la CSP des parents et de l'attention auditive ne sont plus significatives, alors qu'elles l'étaient dans les modèles précédents. Enfin, l'introduction du dernier bloc de variables, celui lié aux compétences des élèves dans le domaine numérique (étape 4), permet à lui seul d'ajouter $29 \%$ à l'explication de la variance totale des performances en résolution de problème. Les performances en calcul mental, en lecture de nombres, en calcul mental avec lexique oral et en base 10 prédisent significativement le niveau en résolution de problèmes. Plus les performances des élèves à ces quatre épreuves augmentent meilleures sont les scores en résolution de

\footnotetext{
${ }^{6}$ Lorsque les variables socio-démographiques sont introduites à l'étape 4 (après toutes les autres variables), les résultats du modèles 4 ne sont pas modifiés.
} 
problèmes. En revanche, les performances aux épreuves de dictée de nombre, de chaîne verbale, de transcodage et en opération n'ont pas d'impact significatif sur ce niveau. Dans ce dernier modèle (modèle 4) qui permet de rendre compte de $70 \%$ de la variance, le score en épreuve de matrices est la seule variable des blocs 1, 2 et 3 à conserver une contribution significative à l'explication de la variance totale.

\section{DISCUSSION}

L'objectif de cette recherche était double. Il s'agissait d'abord de recueillir les performances d'élèves typiques scolarisés au début du collège (en sixième et cinquième) à une série de problèmes arithmétiques verbaux élaborés et testés par ailleurs (Billard, Mirassou \& Touzin, 2019). Il s'agissait ensuite de rechercher par le biais d'indices socio-démographiques et d'épreuves testant des capacités soit générales (langage, mémoire à court terme, etc...) soit spécifiquement liées au domaine numérique les variables ayant ou non une contribution significative sur les scores en résolution de problèmes arithmétiques verbaux et de déterminer leurs poids relatifs.

De manière générale, les résultats antérieurs, portant le plus souvent sur des populations plus jeunes que celles ici retenues, avaient mis en évidence un impact des situations socioéconomiques, les élèves issus de milieux défavorisés obtenant généralement des résultats plus faibles que ceux émanant des classes moyennes ou favorisées (Ursache \& Noble, 2016). Des différences ont également été rapportées relativement au genre, les garçons réussissant le plus souvent mieux que les filles en mathématiques (DEPP, 2019). Nos propres résultats sont conformes à ces données antérieures : les variables démographiques extraient $11 \%$ de la variance. Toutefois, leur poids ne permet pas de déterminer la manière dont elles interviennent sur les performances; il faudrait pour cela s'attacher à des dimensions soit cognitives soit relatives au fonctionnement cérébral, comme certains travaux l'ont déjà fait en recourant à des 
études longitudinales (Noble, Houston, Kan, \& Sowel, 2012 ; Qin, Cho, Rosenberg-Lee, Geary, \& Menon, 2016), ce qui n'a pas été possible dans la présente recherche.

L'introduction des variables associées aux capacités générales non verbales puis verbales contribue pour respectivement $19 \%$ et $11 \%$ de variance aux résultats en résolution de problèmes. De manière intéressante, les dimensions linguistiques font « disparaître » l'impact des dimensions socio-démographiques, suggérant ainsi que ces dernières sont médiatisées par la langage. Là encore, ce résultat est en accord avec ceux de travaux antérieurs, mais seule une approche longitudinale permettrait de l'attester. Quant aux dimensions non verbales, l'attention, souvent mise en avant pour son influence sur les performances (McClelland, Acock, Piccinin, Rhea, \& Stallings, 2013 ; Pagani, Fitzpatrick, Archambault, \& Janosz, 2010) contribue modestement dans le modèle 2 puis son impact s'estompe et devient non significatif. Par contraste, les performances à l'épreuve des matrices construite sur le modèle des matrices de Raven et mettant en jeu le raisonnement déductif et inductif, conservent un impact important même après introduction des variables spécifiquement mathématiques et numériques. Cette persistance d'effet est conforme aux résultats rapportés par Geary (2011) à l'issue d'une étude longitudinale portant sur cinq années : l'intelligence (mesurée par le Raven) reste le meilleur prédicteur des performances ultérieures, au-delà de l'influence de facteurs tels que la vitesse de traitement et de la mémoire de travail.

L'introduction en dernier des dimensions associées aux mathématiques dans la régression hiérarchique visait à en tester l'impact spécifique. Notre raisonnement était que, en accord avec les récents résultats rapportés par Geary, Nicholas, Li, et Sun (2017), l'évolution des performances en mathématiques dépend beaucoup initialement des capacités générales (e.g. attention, mémoire à court terme, etc...) puis, au fur et à mesure que les élèves avancent dans leur scolarité, des savoirs et savoir-faire spécifiques, c'est-à-dire associés à un domaine disciplinaire précis. En nous attachant aux performances d'élèves de classes de sixième et de 
cinquième, nous attendions que le poids des variables spécifiquement mathématiques devienne majoritaire et contribue fortement à la variance totale au-delà de l'impact de toutes les autres variables. Tel est bien le cas puisque la contribution totale des différentes épreuves mathématiques ajoute $29 \%$ de variance aux contributions antérieures de la régression, le modèle total expliquant $70 \%$ de la variance. Le détail des contributions des épreuves fait apparaître qu'à ce niveau de la scolarité, certaines dimensions n'ont plus qu'un poids négligeable (e.g. la chaîne verbale, l'écriture des nombres) alors que d'autres contribuent fortement aux variations de performances : le calcul mental oral ; le calcul écrit ; la maîtrise de la base 10.

Ces données permettent de disposer d'informations nouvelles concernant les performances d'élèves de 11 à 13 ans scolarisés en début de collège. Elles mettent en évidence l'importance à ce niveau des savoirs et savoir-faire spécifiquement mathématiques, plus que celle des capacités générales voire des dimensions démographiques. Certes, ces dernières conservent un poids non négligeable mais la réussite aux résolutions de problèmes est désormais dépendante des acquisitions, notamment relativement à la base 10 (i.e. pour le traitement des décimaux) et aux calculs à l'oral et à l'écrit. Ces données ouvrent deux perspectives, l'une concernant les évaluations - il apparait important de diagnostiquer les niveaux de performance permettant aux élèves de faire face aux exigences de la résolution de problèmes dans le début du second degré - l'autre les interventions. Il est envisageable de concentrer les enseignements sur les questions les plus pertinentes pour la réussite, par exemple en faisant travailler de manière spécifique les dimensions qui sont à la fois les plus fragiles et les plus importantes pour la réussite ultérieure.

Les apports de cette recherche ne doivent pas masquer les limites du travail réalisé. De fait, le recours à des évaluations individuelles de plus de deux cent cinquante élèves mobilise un temps et un personnel considérables. Il s'ensuit que le nombre d'épreuves retenues a dû être réduit, par exemple en restreignant les tests relatifs aux capacités générales et le nombre d'items 
dans certaines épreuves. À ce jour, aucune recherche d'ampleur ne peut couvrir l'ensemble des capacités : c'est la mise en relation avec les résultats d'autres recherches qui permet de faire ressortir les conclusions les plus importantes (i.e. ici avec les données rapportées par Geary, entre autres). Une autre limite tient à l'approche transversale que nous avons adoptée. Les conclusions auxquelles nous avons abouti restent pour certaines spéculatives, par exemple lorsque nous interprétons l'estompage de l'impact des facteurs socio-démographiques par la prise en compte des dimensions relatives aux compétences liées au langage. Il est clair qu'une recherche longitudinale serait seule en mesure d'attester une telle conclusion. Elle devrait être conduite, notamment parce qu'elle permettrait d'établir des trajectoires d'évolution et donc de mieux estimer les relations causales potentielles entre variables, préparant ainsi la mise en place de recherche interventionnelles, seules en mesure de préciser les influences causales. 


\section{BIBLIOGRAPHIE}

Arnold, D. H., \& Doctoroff, G. L. (2003). The early education of socioeconomically disadvantaged children. Annual Review of Psychology, 54, 517-545.

Bagnoud, J., Burra, N., Oakhill, J., \& Thevenot, C. (2018). Arithmetic word problems describing discrete quantities: E.E.G evidence for the construction of a situation model. Acta Psychologica, 190, 116-121.

Billard, C., Mirassou, A., \& Touzin, M. (2019). BMT-i. Paris : Ortho-Édition

Bradley, R. H., \& Corwyn, R. F. (2002). Socioeconomic status and child development. Annual Review of Psychology, 53, 371-399.

Brissiaud, R., \& Sander, E. (2010). Arithmetic word problem solving: A situation strategy first framework. Developmental Science, 13(1), 92-107.

Carpenter, T. P., Hiebert, J.J, \& Moser, M. (1983). The effect of instruction on children's solutions of addition and subtraction word problems. Educational Studies in Mathematics, 14 (1), 55-72.

Coquin-Viennot, D., \& Moreau, S. (2003). Highlighting the role of the episodic situation model in the solving of arithmetical problems. European Journal of Psychology of Education, 18, 267279. 
Cummins, D., Kintsch, W., Reusser, K., \& Weimer, R. (1988). The role of understanding in solving word problems. Cognitive Psychology, 20, 405-438.

Davis-Dorsey, J., Ross, S. M., \& Morrison, G. R. (1991). The role of rewording and context personalization in the solving of mathematical word problems. Journal of Educational Psychology, 83, 61-68.

De Corte, E., Verschaffel, L., \& de Win, L. (1985). Influence of rewording verbal problems on children's problem representations and solutions. Journal of Educational Psychology, 77(4), 460-470.

De Corte, E., \& Verschaffel, L. (1987). The effect of semantic structure on first grader's strategies for solving addition and subtraction word problems. Journal for Research in Mathematics Education, 18, 363-381.

DEPP (2019 mars). L'évolution des performances en calcul des élèves de CM2 à trente ans d'intervalle (1987-2017). Note d'information $\mathrm{n}^{\circ} 19.08$.

De Vos, T. (1992). Tempo Test Rekenen. Nijmegen : Berkhout.

Dixon, P. (1987). The processing of organizational and component step information in written directions. Journal of Memory and Language, 26, 24-35.

Fayol, M., Abdi, H., \& Gombert, J.E. (1987). Arithmetical problems formulation and working memory load. Cognition and Instruction, 4 (3), 183-202. 
Fayol, M., Barrouillet, P., \& Renaud, A. (1996). Pourquoi l'écriture des grands nombres estelle aussi difficile? Revue de Psychologie de l'Education, 1, 109-132.

Fayol, M., Camos, V., \& Roussel, J-L. (2000). Acquisition et mise en œuvre de la numération par les enfants de 2 à 9 ans. In M. Pesenti et X. Seron (Eds.). Neuropsychologie du calcul et du traitement des nombres (p. 33-58). Marseille : Solal.

Fayol, M., Thevenot, C., \& Devidal, M. (2005). La résolution de problèmes arithmétiques verbaux. In M.P. Noël (Ed.). La dyscalculie : Trouble du développement numérique de l'enfant, (p.193-221). Marseille : Solal.

Fischer, J-P, Vilette, B., Joffredo-Lebrun, S., Morellato, M., LeNormand, C., Scheibling-Seve, C., \& Richard, J-F (2019). Should we continue to teach standard written algorithms for the arithmetical operations? The example of subtraction. Educational Studies in Mathematics, 101, 105-121.

Friso-van den Bos, I., van der Ven, S. H. G., Kroesbergen, E. H., \& Van Luit, J. E. H (2013). Working-memory and mathematics in primary school children : A meta-analysis. Educational Research Review, 10, 29-44.

Fuchs, L.S., Fuchs, D., Compton, D.L., Powell, S.R., Seethaler, P.M., Capizzi, A.M., Schatschneider, C., \& Fletcher, J.M. (2006). The cognitive correlates of third-grade skill in arithmetic, algorithmic computation, and arithmetic word problems. Journal of Educational Psychology, 98, $29-43$. 
Fuchs, L.S., Fuchs, D., Stuebing, K., Fletcher, J.M., Hamlett, C.L., \& Lambert, W.E. (2008). Problem solving and computational skill: Are they shared or distinct aspects of mathematical cognition? Journal of Educational Psychology, 100 (1), 30 - 47.

Gamo, S., Taabane, L., \& Sander, E. (2011). Rôle de la nature des variables dans la résolution de problèmes additifs complexes. L'Année Psychologique, 111 (4), 613-640.

Geary, D. C. (2011). Cognitive predictors of achievement growth in mathematics: A five year longitudinal study. Developmental Psychology, 47(6), 1539-1552.

Geary, D. C., Nicholas, A., Li, Y., \& Sun, J. (2017). Developmental changes in the influence of domain-general abilities and domain-specific knowledge on mathematics achievement. An eight-year longitudinal study. Journal of Educational Psychology, 109 (5), 680-693.

IEA. (2015). TIMSS 2015 International Database webpage https://timssandpirls.bc.edu/timss2015/advanced-international-database/

INSEE. (2003). Nomenclature des professions et catégories socioprofessionnelles. Paris : INSEE.

Jarlégan, A., Fayol, M., \& Barrouillet, P. (1996). De soixante-douze à 72 et inversement : Une étude du transcodage chez les enfants de sept ans. Revue de Psychologie de l'Education, 1, 87108. 
Jimėnez, L., \& Verschaffel, L. (2014). Development of children's solutions of non-standard arithmetic word problem solving. Revista de Psicodidáctica, 19, 93-123.

Johnson-Laird, P. N. (1983). Mental models: Towards a cognitive science of language, inference, and consciousness. Cambridge, MA: Harvard University Press.

Jordan, N. C., \& Hanich, L. B. (2000). Mathematical thinking in second-grade children with different forms of LD. Journal of Learning Disabilities, 33, 567 - 578.

Jordan, N. C., Huttenlocher, J., \& Levine, S. C. (1992). Differential calculation abilities in young children from middle-and low-income families. Developmental Psychology, 28 (4), 644 653.

Jordan, N. C., Kaplan, D., \& Hanich, L. B. (2002). Achievement growth in children with learning difficulties in mathematics: Findings of a two-year longitudinal study. Journal of Educational Psychology, 94 (3), 586-597.

Jordan, N. C., Levine, S. C., \& Huttenlocher, J. (1994). Development of calculation abilities in middle- and low-income children after formal instruction in school. Journal of Applied Developmental Psychology, 15(2), 223-240.

Kail, R., \& Hall, L. K. (1999). Sources of developmental change in children's word-problem performance. Journal of Educational Psychology, 91(4), 660-668.

Kintsch, W., \& Greeno, J. G. (1985). Understanding and solving word arithmetic problems. 
Psychological Review, 92, 109-129.

Kline, R. B. (1998). Methodology in the social sciences. Principles and practice of structural equation modeling. New York, NY, US: Guilford Press.

Levine, S., Jordan, N.C., \& Huttenlocher, J. (1992). Development of calculation abilities in young children. Journal of Experimental Child Psychology, 53, 72-103.

Lewis, A., \& Mayer, R. E. (1987). Students' miscomprehension of relational statements in arithmetic word problems. Journal of Educational Psychology, 79, 363-371.

Lortie-Forgues, H., Tian, J., \& Siegler, R. S. (2015). Why is learning fraction and decimal arithmetic so difficult? Developmental Review, 38, 201-221.

McClelland, M. M., Acock, A. C., Piccinin, A., Rhea, S. A., \& Stallings, M. C. (2013). Relations between preschool attention span-persistence and age 25 educational outcomes. Early Childhood Research Quarterly, 28, 314-324.

Ménissier, A. (2011). Analyser, comprendre et travailler avec les problèmes arithmétiques. In M. Habib, M-P. Noël, F. George et V. Brun (eds.). Calcul et dyscalculies : des modèles à la rééducation, (p.79-129). Issy-les-Moulineaux : Elsevier Masson.

Muth, K.D. (1994). Solving arithmetic word problems: Role of reading and computational skills. Journal of Educational Psychology, 76 (2), 205-210. 
Noble, K. G., Houston, S. M., Kan, E., \& Sowell, E. R. (2012a). Neural correlates of socioeconomic status in the developing human brain. Developmental Science, 15, 516-527.

OECD. (2015). About PISA. Retrieved from http://www.oecd.org/pisa/aboutpisa/

Pagani, L. S., Fitzpatrick, C., Archambault, I., \& Janosz, M. (2010). School readiness and later achievement : A French Canadian replication and extension. Developmental Psychology, 46 (5), 984-994.

Peng, P., Namkung, J., Barnes, M., \& Sun, C. (2016). A meta-analysis of mathematics and working memory: Moderating effects of working memory domain, type of mathematics skill, and sample characteristics. Journal of Educational Psychology, 108 (4), 455-473.

Pimperton, H., \& Nation, K. (2010). Understanding words, understanding numbers: an exploration of the mathematical profiles of poor comprehenders. British Journal of Educational Psychology, 80 (2), 255-268.

Purpura, D. J., \& Reid, E. E. (2016). Mathematics and language : Individual and group differences in mathematical language skills in young children. Early Childhood Research Quarterly, 36, 259-268.

Qin, S., Cho, S., Rosenberg-Lee, M., Geary, D. C., \& Menon, V. (2015). Hippocampalneocortical functional reorganization underslies children's cognitive development. Nature Neurosciences, 17 (9), 1263-1269. 
Raghubar, K. P., Barnes, M. A., \& Hecht, S. A. (2010). Working memory and mathematics: A review of developmental, individual differences, and cognitive approaches. Learning and Individual Differences, 20, 110-122.

Rosenthal, D. J. A., \& Resnick, L. B. (1974). Children's solution processes in arithmetic word problems. Journal of Educational Psychology, 66(6), 817-825.

Schumacher, R. F., \& Fuchs, L.S., (2012). Does understanding relational terminology mediate effects of intervention on compare word problems? Journal of Experimental Child Psychology, $111,607-28$

Siegler, R. S., \& Pyke, A. A. (2013). Developmental and individual differences in understanding of fractions. Developmental Psychology, 49 (10), 1994-2004.

Stern, E., \& Lehrndorfer, A. (1992). The role of situational context in solving word problems. Cognitive Development, 7 (2), 259-268

Swanson, H. L. (2011). Working memory, attention, and mathematical problem solving: A longitudinal study of elementary school children. Journal of Educational Psychology, 103 (4), $821-837$

Swanson, H.L., Cooney, J.B., \& Brock, S. (1993). The influence of working memory and classification ability on children's word problem solution. Journal of Experimental Child Psychology, 55, 374-395. 
Swanson, H. L., Olide, A. F., \& Kong, J. E. (2018). Latent class analysis of children with math difficulties and/or math learning disabilities: Are there cognitive differences? Journal of Educational Psychology, 110 (7), 931-951.

Tarim, K. (2017). Problem solving levels of elementary school students on mathematical word problems and the distribution of these problems in textbooks. Cukurova University Faculty of Education Journal, 46, 639-648.

Thevenot, C. (2008). Fifth grader's mental representation and strategies for solving arithmetic word problem. L'Année Psychologique, 108, 617-630.

Thevenot, C. (2010). Arithmetic word problem solving: Evidence for the construction of a mental model. Acta Psychologica, 133, 90-95.

Thevenot, C. (2017). Arithmetical word problem solving: The role of prior knowledge. In D. Geary, D. Berch, K. Mann-Koepke (Eds.). Mathematical Cognition and Learning. Vol.3: Acquisition of Complex Arithmetic Skills and Higher-Order Mathematics Concepts, (p. 47-66). Academic Press.

Thevenot, C., \& Barrouillet, P. (2015). Word problem solving and mental representations. In A. Dowker, \& R. Cohen-Kadosh (Eds.). The Oxford Handbook of Numerical Cognition, (p. 158-179). Oxford University Press. 
Thevenot, C., Devidal, M., Barrouillet, P., \& Fayol, M. (2007). Why does placing the question before an arithmetic word problem improve performance? A situation model account. Quarterly Journal of Experimental Psychology, 60 (1), 43-56.

Tolar, T. D., Fuchs, L., Cirino, P. T., Fuchs, D., Hamlett, C. L., \& Fletcher, J. M. (2012). Predicting development of mathematical word problem solving across the intermediate grades. Journal of Educational Psychology, 104(4), 1083-1093.

Ursache, A., \& Noble, K. G. (2016). Neurocognitive development in socioeconomic context : Multiple mechanisms and implications for measuring socioeconomic status. Psychophysiology, $53,71-82$.

Van Dijk, T., \& Kintsch, W. (1983). Strategies of discourse comprehension. New York: Academic Press.

Van Dooren, W., Lehtinen, E., \& Verschaffel, L. (2015). Unraveling the gap between natural and rational numbers. Learning and Instruction, 37, 1-4.

Verschaffel, L., De Corte, E., \& Lasure, S. (1994). Realistic considerations in mathematical modeling of school arithmetic word problems. Learning and Instruction, 4, 273-294.

Willis, G. B., \& Fuson, K. C. (1988). Teaching children to use schematic drawings to solve addition and subtraction word problems. Journal of Educational Psychology, 80(2), 192-201. 
Wang, A. Y., Fuchs, L., S., \& Fuchs, D. (2016). Cognitive and linguistic predictors of mathematical word problems with and without irrelevant information. Learning and Individual Differences, 52, 79-87. 
Annexe 1 : Matrice de corrélations entre les différentes variables de l'étude

\begin{tabular}{|c|c|c|c|c|c|c|c|c|c|c|c|c|c|}
\hline & 1 & 2 & 3 & 4 & 5 & 6 & 7 & 8 & 9 & 10 & 11 & 12 & 13 \\
\hline 1 & 1 & & & & & & & & & & & & \\
\hline 2 & $.57^{* *}$ & 1 & & & & & & & & & & & \\
\hline 3 & $.49^{* *}$ & $.35^{* *}$ & 1 & & & & & & & & & & \\
\hline 4 & $.39^{* *}$ & $.46^{* *}$ & $.27^{* *}$ & 1 & & & & & & & & & \\
\hline 5 & $.49^{* *}$ & $.35^{* *}$ & $.58^{* *}$ & $.20^{* *}$ & 1 & & & & & & & & \\
\hline 6 & $.74^{* *}$ & $.59^{* *}$ & $.43^{* *}$ & $.40^{* *}$ & $.36^{* *}$ & 1 & & & & & & & \\
\hline 7 & $.35^{* *}$ & $.34^{* *}$ & $.52^{* *}$ & $.17^{* *}$ & $.51^{* *}$ & $.30^{* *}$ & 1 & & & & & & \\
\hline 8 & $.35^{* *}$ & $.33^{* *}$ & $.30^{* *}$ & $.19^{* *}$ & $.28^{* *}$ & $.30^{* *}$ & $.22^{* *}$ & 1 & & & & & \\
\hline 9 & $.66^{* *}$ & $.48^{* *}$ & $.48^{* *}$ & $.33^{* *}$ & $.47^{* *}$ & $.62^{* *}$ & $.34^{* *}$ & $.34^{* *}$ & 1 & & & & \\
\hline 10 & $.50^{* *}$ & $29^{* *}$ & $.35^{* *}$ & $.21^{* *}$ & $.28^{* *}$ & $.54^{* *}$ & $.18^{* *}$ & $.30^{* *}$ & $.37^{* *}$ & 1 & & & \\
\hline 11 & $36^{* *}$ & $21^{* *}$ & $.22^{* *}$ & .10 & $.15^{*}$ & $.38^{* *}$ & $.14^{*}$ & $.12^{*}$ & $.16^{* *}$ & $.48^{* *}$ & 1 & & \\
\hline 12 & $-.40^{* *}$ & $-.45^{* *}$ & $-.32^{* *}$ & $-.29^{* *}$ & $-.22^{* *}$ & $-.45^{* *}$ & $-.26^{* *}$ & $-.27^{* *}$ & $-.39^{* *}$ & $-.48^{* *}$ & $-.29^{* *}$ & 1 & \\
\hline 13 & $.22^{* *}$ & $.21^{* *}$ & .11 & $.18^{* *}$ & .11 & $.31^{* *}$ & $.13^{*}$ & $.19^{* *}$ & $.19^{* *}$ & $.22^{* *}$ & $.27^{* *}$ & $-.28^{* *}$ & 1 \\
\hline 14 & $.48^{* *}$ & $20^{* *}$ & $.31^{* *}$ & $.19^{* *}$ & $20^{* *}$ & $.43^{* *}$ & $.14^{*}$ & $27^{* *}$ & $37^{* *}$ & $.68^{* *}$ & $.38^{* *}$ & $-.42^{* *}$ & $.27^{* *}$ \\
\hline
\end{tabular}

\section{Annexe 2 : Liste des 20 problèmes arithmétiques verbaux}

Problème $\mathrm{n}^{\circ} 1$ :

Il y a 28 élèves dans la classe. Le professeur a fait des groupes de 7 élèves. Combien y a-t-il de groupes de 7 élèves ? 4 groupes

Problème $\mathrm{n}^{\circ} 2$ :

$4 \mathrm{CD}$ coûtent $12 €$. Combien coûtent $10 \mathrm{CD}$ ? 30 euros

PROBLEME $\mathrm{n}^{\circ} 3$ :

Pour son anniversaire, Clément achète 2 packs de bouteilles de jus de fruit. Il y a 4 bouteilles dans un pack et chaque bouteille coûte $3 €$. Quel est le prix payé par Clément pour son achat ? $\underline{24 \text { euros }}$

PROBLEME $\mathrm{n}^{\circ}$ 4:

Charlotte a dépensé $18 €$ pour acheter un polo. Quand elle règle son achat, la vendeuse lui rend alors $32 €$. Quelle somme d'argent avait-elle donnée pour régler sa dépense ? 50 euros

Problème $\mathrm{n}^{\circ} 5$ :

Plusieurs chatons se sont cachés dans un grand panier. On voit dépasser 10 pattes et 2 queues. Combien y-a-t-il de chatons au minimum ? $\underline{3}$

Problème $n^{\circ}$ 6: 
Pierre a 12 DVD. Il en a 4 fois moins que Jean. Combien Jean a-t-il de DVD ? 48 DVD

Problème $\mathrm{n}^{\circ} 7$ :

Alex a construit une pile de 11 cubes. Manon a construit une pile de 5 cubes. Tous les cubes sont de la même taille et il n'y a pas d'autres cubes. Combien Alex doit-il donner de cubes à Manon pour que les deux piles soient à la même hauteur?

Problème $\mathrm{n}^{\circ} 8$ :

5 cageots de pommes pèsent $30 \mathrm{~kg}$. Combien pèsent 3 cageots ?

PROBLEME $n^{\circ}$ 9:

En France, il y a de longs fleuves. La Garonne a une longueur totale de $650 \mathrm{~km}$, mais elle mesure $130 \mathrm{~km}$ de moins que la Seine. Quelle est la longueur de la Seine?

Problème $n^{\circ} 10$ :

Marie a 3 frères et 2 sœurs. Combien de frères et de sœurs a son frère Michel ?

Problème ${ }^{\circ} 11:$

Au début de la matinée, Florian possédait 23 billes. Il a joué une première partie dans la matinée. Puis il a joué une seconde partie l'après midi où il a gagné 9 billes. A la fin de la journée, Florian a 27 billes. Combien a t-il gagné ou perdu de billes au cours de la première partie de la matinée ?

\section{PROBLEME $\mathrm{n}^{\circ} 12$ :}

Le prix du camping municipal est de $4 €$ par personne et par jour. Une famille de 5 personnes a séjourné dans le camping durant 1 semaine. Quelle a été la dépense totale de cette famille ?

PROBLEME $n^{\circ} 13$ :

Une bougie d'anniversaire met 12 minutes pour brûler dans sa totalité. Combien de temps mettront les 8 bougies du gâteau d'anniversaire de Manon si on les allume toutes en même temps?

\section{PROBLEME $\mathrm{n}^{\circ} 14$ :}

Julie a acheté 3 petits pains et 2 brioches pour $4 €$. Léa achète 9 petits pains et 6 brioches. Combien Léa paiera-t-elle pour son achat?

\section{PROBLEME $n^{\circ} 15$}

Dans une potion préparée par Harry Potter, il y a $12 \%$ de miel.

La potion pèse $50 \mathrm{~g}$. Quelle est la masse de miel ?

$$
\begin{aligned}
& 24 \mathrm{~g} \\
& 12 \mathrm{~g} \\
& 6 \mathrm{~g} \\
& 3 \mathrm{~g}
\end{aligned}
$$

$\underline{\text { Problème } \mathrm{n}^{\circ} 16}$ :

Ce matin, j'ai prêté 15 DVD à Léa et puis Jules m'a rendu 9 autres DVD. Maintenant, j'ai 20 DVD.

Combien en avais-je tout au début, avant d'en prêter ? 
Problème $\mathrm{n}^{\circ} 17$ :

Thomas achète un jeu vidéo en solde car il le paye les $2 / 3$ de son prix. Avant, le jeu vidéo valait $36 €$. Combien Thomas a-t-il payé son jeu vidéo?

\section{PROBLEME $n^{\circ} 18$}

Sarah achète 3 pots de peinture à $18 €$ chacun, et 2 pinceaux. Elle paie avec un billet de $100 €$. On lui rend $40 €$ de monnaie. Combien coûte chaque pinceau ?

Problème $\mathrm{n}^{\circ} 19$ :

Il y a 24 élèves dans la classe. Il y a deux fois plus de filles que de garçons. Combien y a-t-il de garçons dans la classe?

Problème $\mathrm{n}^{\circ} 20$ :

Dans une course, si tu dépasses le second, quelle sera ta place? 